\section{CASE REPORT}

\author{
M. Ozaki \\ Y. Murayama \\ M. Ebara \\ H. Takao \\ T. Abe
}

\title{
Delayed Hydrocephalus after Embolization of Unruptured Aneurysms Using Bare Platinum Coils: Report of 2 Cases
}

SUMMARY: Postembolization hydrocephalus with bare platinum coils has not been reported, to our knowledge. We report 2 cases of unusual delayed hydrocephalus after treatment of unruptured intracranial aneurysms with bare platinum coils.

ABBREVIATIONS: DSA = digital subtraction angiography; $\mathrm{GDC}=$ Guglielmi detachable coil; ICA = internal carotid artery; MRA = MR angiography; PcomA = posterior communicating artery; $\mathrm{V}-\mathrm{P}=$ ventricular-peritoneal

A septic meningitis, hydrocephalus, and perianeurysmal edema are being increasingly reported in patients treated with surface-modified coils such as hydrogel-coated coils. ${ }^{1-4}$ However, postembolization delayed hydrocephalus with bare platinum coils has not been reported. We report 2 cases of unusual delayed hydrocephalus after treatment with bare platinum coils.

\section{Case Reports}

\section{Case 1}

A 69-year-old woman presented with an incidentally discovered right ICA PcomA aneurysm that measured $15 \mathrm{~mm}$ on DSA (Fig 1). No perianeurysmal edema or hydrocephalus was seen on the initial MR imaging. GDC (Boston Scientific, Natick, Massachusetts) embolization was performed by using a balloon-assisted technique. There were no technical complications, including subarachnoid hemorrhage. Postembolization angiography demonstrated a small neck remnant. The patient was discharged without symptoms. An MRA 1 year later revealed aneurysm recurrence. Two additional coil embolizations were required.

Approximately 18 months after the third embolization, the patient presented with symptoms of dementia and impaired coordination. MR imaging showed hydrocephalus (Evans index, 0.32), and there was no evidence of mechanical obstruction of CSF pathways by the coil mass. A V-P shunt surgery was performed, and CSF findings showed no evidence of meningitis. The patient recovered completely.

\section{Case 2}

A 66-year-old woman presented with an incidentally discovered left paraclinoid ICA aneurysm measuring $22 \mathrm{~mm}$ on DSA (Fig 2). There was no perianeurysmal edema on the initial MR imaging. GDC embolization was performed with a small neck remnant, and the patient was discharged without complications.

One-year follow-up MRA revealed coil compaction and aneurysm

Received July 12, 2010; accepted after revision August 4

From the Department of Neurosurgery, Division of Endovascular Neurosurgery, The Jikei University School of Medicine, Tokyo, Japan.

Y. Murayama, MD, is an inventor of the Matrix detachable coil (Boston Scientific).

Please address correspondence to Yuichi Murayama, MD, Division of Endovascular Neurosurgery, Department of Neurosurgery, The Jikei University School of Medicine, 3-25-8 Nishi-Shinbashi, Minato-ku, Tokyo, Japan; e-mail: ymurayama@jikei.ac.jp

http://dx.doi.org/10.3174/ajnr.A2317 recurrence. An additional coil embolization was performed, and postembolization cerebral angiography demonstrated a small remnant. There were no technical complications throughout the procedures.

Approximately 18 months after the second embolization, the patient presented with symptoms of dementia and impaired coordination. An MR image showed hydrocephalus (Evans index, 0.34), and there was no evidence of mechanical obstruction of CSF pathways by the coil mass. CSF examination findings were within normal limits. A V-P shunt was placed, and the patient was discharged without symptoms.

\section{Discussion}

Hydrocephalus after the treatment of unruptured intracranial aneurysms is rare. No cases after treatment by clipping have been reported, but several reports described the detection of hydrocephalus after coiling with hydrogel coils. ${ }^{1-4}$

To our knowledge, our observation is the first report of hydrocephalus after bare platinum coil embolization for unruptured aneurysms. In ruptured aneurysms, however, White et $\mathrm{al}^{5}$ reported the presence of hydrocephalus, but the contribution of pre-existing subarachnoid hemorrhage could not be excluded. ${ }^{5}$

The phenomena behind the development of hydrocephalus remain unclear. Different hypotheses have been proposed to relate the procedure and particularly materials used to the complication. With bare platinum coils, mechanical obstruction of the CSF pathway due to the expansion of the embolized coil mass can be 1 cause of postembolization hydrocephalus. However, our 2 cases of ICA aneurysms were not related to the ventricular outflow CSF pathways. There were no clear etiology and pathogenesis for these cases.

The effect of embolic material on the aneurysm wall and beyond it has also been a concern. Aneurysm wall enhancement and perianeurysmal edema have been detected after the use of different coil types-18.6\% in bare platinum, $25.7 \%$ in Matrix (Boston Scientific), and $81.8 \%$ in hydrogel coils. ${ }^{4}$ If the mechanisms of wall enhancement and perianeurysmal edema and hydrocephalus are related, it seems logical to expect, even to a lesser extent, the presence of hydrocephalus after coiling with coil types other than hydrogel. However, in the series of Fanning et al, ${ }^{4}$ the cases with hydrocephalus did not have perianeurysmal edema. 

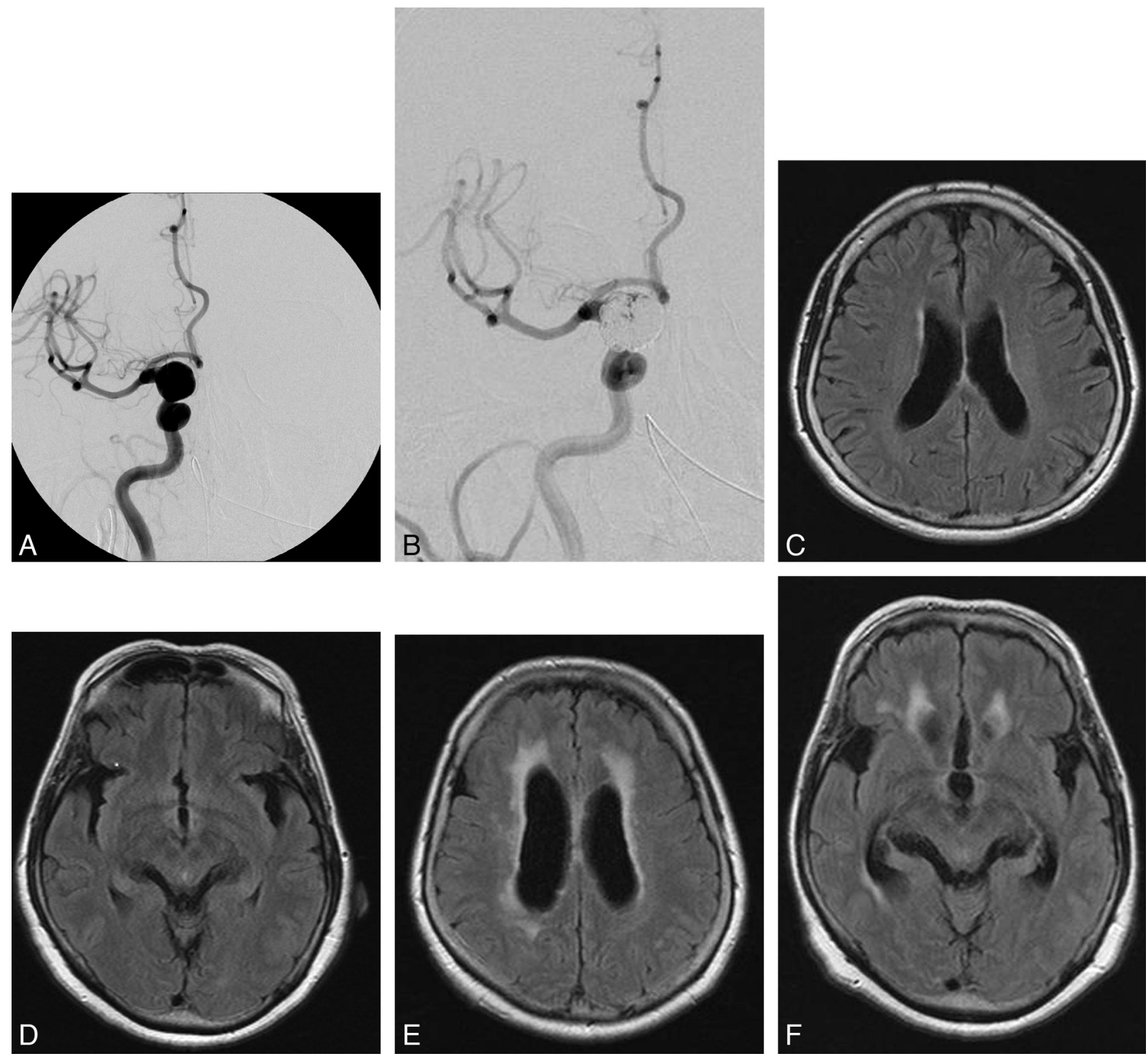

Fig 1. A 69-year-old woman with a large right ICA PcomA aneurysm. A, Pre-embolization angiogram shows a 15-mm ICA PcomA aneurysm (lateral view). B, After the third embolization of the aneurysm, there is a neck remnant. $C$ and $D$, Pre-embolization MR images show no hydrocephalus. There is no evidence of mechanical obstruction of CSF pathways with the coil mass. $E$ and $F$, Eighteen months after the third embolization (48 months after the initial embolization), MR images show hydrocephalus.

Some other factors or mechanisms causing hydrocephalus can be explored, such as size, location, age of patient, and so forth, and ultimately multiple factors and mechanisms might be responsible. For that reason, we looked at the complete case series of bare platinum coil embolizations of our institution of 512 unruptured aneurysms, including the 2 reported cases, in which the average age and size was 68 years and $18.5 \mathrm{~mm}$, respectively; the hydrocephalus incidence was $0.4 \%$. Our 2 patients required multiple coil embolizations, and aneurysms were in closer contact with the brain parenchyma on MR imaging. Therefore, 1 reason might be a chronic inflammatory response developed with incomplete occlusion of the aneurysms. Long-term water-hammer effect might also have caused a brain parenchymal inflammatory response. On the other hand, a possible mechanism for the cause of hydroceph- alus with hydrogel-coated coils may be chemical components of hydrogel polymer or an associated breakdown product of the CSF.

\section{Conclusions}

This report demonstrates that hydrocephalus can occur after treatment of large unruptured intracranial aneurysms with bare platinum coils, even though it is a rare complication. Although the incidence is extremely low, hydrocephalus appears to be associated with the coiling itself and not only related to the presence of hydrogel polymer or another specific factor that may increase its rate. Patients with large aneurysms embedded into the brain parenchyma might carry a certain risk of developing delayed hydrocephalus after coil embolization, even with bare platinum coils. 

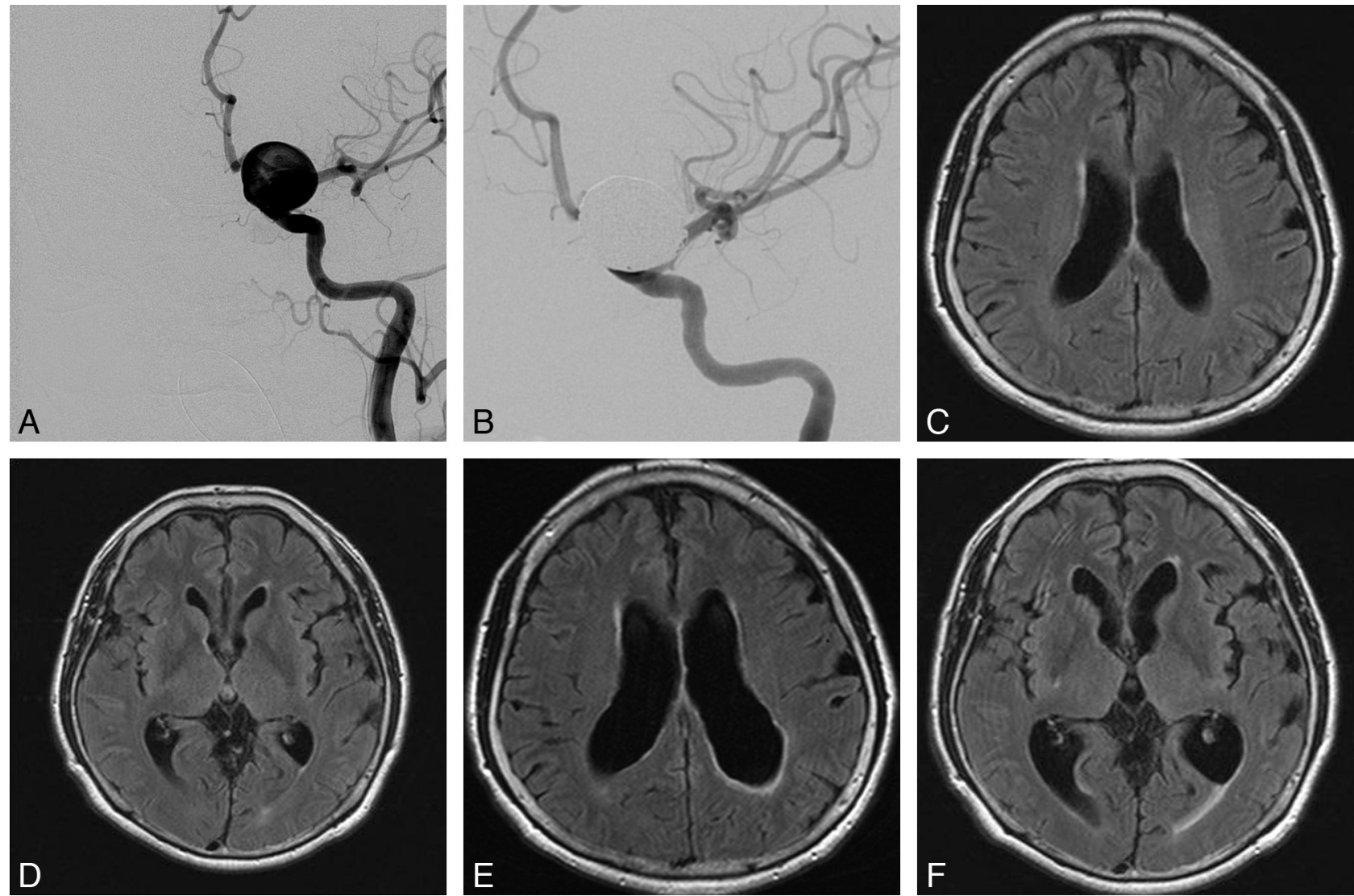

Fig 2. A 66-year-old woman with a large ICA paraclinoid aneurysm. $A$, Pre-embolization angiogram shows a 22-mm ICA paraclinoid aneurysm. $B$, Postembolization angiogram shows near-complete occlusion. $C$ and $D$, Pre-embolization MR images (fluid-attenuated inversion recovery) show no hydrocephalus. $E$ and $F$, Eighteen months after the second embolization (30 months after the initial embolization), MR images show hydrocephalus, and no evidence of mechanical obstruction of CSF outflow pathways with the coil mass.

\section{In Memoriam}

In the final stage before submitting this manuscript, our brilliant resident, dedicated with his whole heart to neurosurgery, first author of the article, Dr. Ozaki, unexpectedly passed away. This was his last work, and its submission to the American Journal of Neuroradiology is an appreciation and a tribute to the effort of his life, interrupted so early.

\section{Acknowledgments}

We thank Kostadin Karagiozov, MD, for his preparation of this manuscript.

\section{References}

1. Berenstein A, Song JK, Niimi Y, et al. Treatment of cerebral aneurysms with hydrogel coated platinum coils (HydroCoil): early single-center experience. AJNR Am J Neuroradiol 2006;27:1834-40

2. Kang HS, Han MH, Lee TH, et al. Embolization of intracranial aneurysms with hydrogel-coated coils: result of a Korean multicenter trial. Neurosurgery 2007;61:51-58

3. Marchan EM, Sekula RF Jr, Ku A, et al. Hydrogel coil-related delayed hydrocephalus in patients with unruptured aneurysms. J Neurosurg 2008;109:186-90

4. Fanning NF, Willinsky RA, terBrugge KG. Wall enhancement, edema, and hydrocephalus after endovascular coil occlusion of intradural cerebral aneurysms. J Neurosurg 2008;108:1074-86

5. White JB, Cloft HJ, Kallmes DF. But did you use HydroCoil? Perianeurysmal edema and hydrocephalus with bare platinum coils. AJNR Am J Neuroradiol 2008;29:299-300 